\title{
Advances in Molecular Pathways and Targeted Therapy of Colon Cancer
}

\author{
Xiaochuang Si, Yanni Gu, Nan Meng, Guannan Yang, Xuepeng Zhang* \\ Affiliated Hospital of North China University of Science and Technology \\ *Correspondence Author
}

\begin{abstract}
Colon cancer is the third largest malignant tumor in the world and the fourth leading cause of cancer death. According to statistics, there are about 2 million newly diagnosed patients every year[1].The etiology and pathogenesis of colon cancer have not been completely clear, and it is believed that it is mainly the result of comprehensive action of organism internal cause and environment external cause. Research reports, the occurrence of colorectal cancer may be more outcome of combined action of a gene mutation, abnormal changes of the disease involving multiple genes and chromosomes, including both proto-oncogene ras and myc activation, APC and tumor suppressor genes, the inactivation of DCC, MCC and p53, etc, also and way to chromosome instability, such as chromosome number widely disorders and loss of heterozygosity[2]. The occurrence and development of colorectal cancer involves gene polymorphism of metabolic enzyme. In a sense, colorectal cancer is a genetic disease caused by gene mutation. On the other hand, the occurrence and development of colorectal cancer and lifestyle, dietary factors, intestinal flora, previous benign history of large intestine and mental stimulation and other environmental factors are also closely related, therefore, the study of its molecular pathway for the diagnosis and treatment of colon cancer has important guiding significance.
\end{abstract}

Keywords: Colon cancer, Molecular signaling pathway, Colon cancer inhibitors.

\section{Introduction}

Implementation of early screening, advances in surgical techniques and personalized chemotherapy drugs have significantly reduced mortality and morbidity in colon cancer patients, Despite advances in colon cancer prevention and early diagnosis, disease-free survival rates for patients diagnosed with advanced colon cancer remain very low, with more than 800,000 colon cancer patients dying from this malignant disease in 2018 alone[3]. Colon cancer has unique clinical, molecular, and pathophysiological features, and a better understanding of the occurrence and progression of CRC could help develop early detection of molecular markers and risk stratification methods to improve clinical care for colon cancer patients. Traditionally, subgroups of colon cancer patients have been associated with KRAS, or BRAF mutations (chromosomal instability group); Mismatch repair gene dysfunction (microsatellite instability group, MSI); And hypermethylation $(\mathrm{CpG}$ island methylation phenotype, CIMP)[4].

\section{Molecular Basis of Colon Cancer}

\subsection{Chromosomal Instability Group (CIN)}

Chromosomal instability refers to a significant increase in gain or loss of all or most chromosomes and is the most common genetic instability in colon cancer. Chromosomal instability group (CIN) exists in about $85 \%$ of adenocarcinoma transformation and is characterized by activation of oncogenes (KRAS and BRAF), inactivation of TSG (APC and TP53) and loss of heterozygosis in the long arm of chromosome $18(18 \mathrm{q} \mathrm{LOH})$, thereby promoting the occurrence and development of colon cancer tumors[5].

\subsubsection{Wnt/ $\beta$-catenin Signaling Pathway}

The Wnt signaling pathway regulates multiple cellular processes, such as cell movement and proliferation during embryonic development. Wnt signaling is also involved in stem cell potency maintenance and differentiation induction. Abnormal Wnt signaling pathway is associated with the development of colon cancer, and the Wnt/ $\beta$-catenin signaling pathway cascaded in the center of cancer development, and about $90 \%$ of colon cancer patients have mutations in this signaling pathway[6]. These mutations occur mainly in colon adenomatous polyposis gene (adenomatouspolyposiscoli, APC) and beta serial protein gene ( $\beta$-catenin), both can lead to abnormal pathways activated, but other pathway components can be mutated. Previous studies have found that APC encodes negative regulatory factors of the Wnt pathway, so APC inactivation can easily activate the Wnt signaling pathway[7]. Meanwhile, Wnt signaling pathway is involved in epithelial-mesenchymal transformation (EMT) by regulating the expression of $\beta$-catenin, and thus is involved in the formation and metastasis of various epithelial tumors, including colon cancer. In colon cancer, EMT affects the proliferation of CCSC, the development of colon cancer, and regulates the degree of malignancy and distal metastasis. In addition, Vermeulen[8] showed that abnormal activation of Wnt signaling pathway can enhance the proliferation and invasion ability of colon cancer cells, which is caused by increased expression of stem cell molecular markers. Lgr5 is a target gene of Wnt signaling pathway and a common surface marker of CSC, suggesting that Wnt signaling pathway can be used as a marker of carcinogenic ability. The $\mathrm{Wnt} / \beta$-catenin signal transduction pathway is at the center of tumor genesis and progression, and anticancer drugs targeting this pathway have great potential. Currently under study, the drugs mainly target Wnt ligand and receptor (LGK974I, OMP-54F28, OMP-18R5 and OTSA101), $\beta$-catenin degradation complex (crecoxib, Dif1/3, dye lignin, Pyrvinium, G007-LK, XAV939, JW55), $\beta$-catenin cytoplasmic nuclear translocus (WGA) and $\beta$-catenin transcription complex (PRI724, Vitamin D, retinoic acid, PKF115-584, CGP9049090 and SAH-BCL9). Among them, membrane-bound O-acyltransferase Porcupine (PORCN) modifies Wnt ligand lipids, promotes its secretion, and enhances its binding ability to homologous receptors. 
LGK974 is an effective specific small-molecule inhibitor of PORCN, targeting colon cancer by down-regulation of Wnt target gene Axin2 expression and reducing phosphorylation of LRP6, and is currently in phase I clinical trial[9]. High expression of cyclooxygenase- 2 (cox-2) can promote the production of prostaglandin E2, and then inhibit the expression of glycogen synthase kinase $3 \beta$ and Axin2 to prevent $\beta$-catenin degradation, and finally activate the Wnt pathway. Nonsteroidal anti-inflammatorydrugs (NSAIDs), such as aspirin, sulinic acid or specific COX-2 inhibitors, such as celecoxib, can inhibit Wnt signal transduction by inhibiting COX-2.In addition, Chen et al.[10] found that FH535 can effectively down-regulate the expression of target genes such as cyclinD1 and Survivin at mRNA and protein levels by targeting the $\mathrm{Wnt} / \beta$-catenin signaling pathway, and inhibit the proliferation of colon cancer cells.

\subsubsection{TP53 Pathway}

TP53 gene is located on the short arm of chromosome 17 and TP53 mutation or loss of function has been reported in $50-75 \%$ of colon cancer cases; Loss of tp53-mediated apoptosis pathway is an important determinant of progression from adenoma to malignant tumor[11]. Enhanced loss of function of the p53 gene, high cell proliferative activity and uncontrolled cell cycle are a key step in the development of colon cancer. Studies have shown that missense mutation $(48 \%)$ is the most common mutation of TP53 in colon cancer[12]. This was followed by point mutation (37.5\%), a shift at the $\mathrm{CpG}$ site. In addition, wild-type p53 has been identified as a direct activator of WAD-1, a gene that highly induces inhibition of tumor cell growth in the P53 pathway[13]. Other mutations may occur during the development and progression of colon cancer[14]. Adenosine monophosphate activated protein kinase (AMPK) pathway induces phosphorylation of p53 and apK-dependent cell cycle arrest, thereby upregulating cyclin-dependent kinase inhibitor 1A (CDKN1A or P21). Ultimately, cell cycle regulation, cell senescence and stem cell senescence are controlled[15].P53 also interacts with cycde-2 (COX-2) and is involved in enhancing inflammation and colon cancer cell proliferation. Interestingly, coX-2 positive tumors were significantly associated with cancer-specific mortality regardless of p53 status, suggesting that COX-2 is an independent prognostic factor for colon cancer[16].

\subsubsection{The 18q Loss of Heterozygosity (LOH)}

Deletion of heterozygosity ( $\mathrm{LOH}$ ) is the deletion of one of two copies or alleles of a gene, and the remaining alleles are often affected by mutations. $\mathrm{LOH}$ in the $18 \mathrm{q}$ region is most common in advanced colon cancer and is associated with poor prognosis of CRC. Colon cancer is located on chromosome $18 \mathrm{q} 21.2$, and the loss of expression of $18 \mathrm{qLOH}$ plays an important role in the pathogenesis of colon cancer. Netrin-1 exists in the crypt of colorectal mucosa. Epithelial cell differentiation leads to the loss of Netrin-1 expression, and gene mutations inhibit the binding of Netrin-1 to cell transmembrane proteins, leading to abnormal cell survival[17]. On the other hand, SMAD2 and SMAD4 are present in 18q21.1, a region commonly lost during colon cancer progression, and are associated with adenoma development and adenocarcinoma progression in mouse models, suggesting that the SMAD gene may have a tumor suppressive effect. In addition, immunohistochemical analysis reported a loss of SMAD4 expression in $>50 \%$ of CRC, which is associated with lymph node metastasis[18]. Studies have shown that the SMAD gene encodes the TGF- $\beta$ protein, and dysregulation of TGF- $\beta$ signaling occurs in most CRC. Functionally, significant mutations of TGF- $\beta$ R2 are present in approximately $30 \%$ of CRC cases and are associated with malignant transformation of advanced adenomas.

\subsection{Microsatellite Instability Group (MSI)}

Another type of genomic instability in colon cancer is microsatellite instability (MSI), which is unique to cancer cells. Tumor subsets with $\geq 30 \%$ marker unstable sites are defined as "microsatellite high" (MSI-H), and tumor subsets with 10-29\% unstable sites are classified as "microsatellite low" (MSI-L). And "microsatellite stability" (MSS) without instability markers. In cancers with MSI-H, small insertions/deletions lead to frameshift mutations within repeated regions of tumor gene coding regions, further promoting tumorigenesis. Large-scale genomic screening of microsatellite coding regions revealed mutations in nine loci (TGF- $\beta$ R2, Bax, MSH3, ActRIIB, SEC63, AIM2, NADH-ubiquinone oxidation-reductase, COBLL1 and EBP1). $>20 \%$ of tumors. TGF- $\beta$ R2 is the most common mutation site, with instability in the TGF- $\beta$ R2 polyadenine bundle present in approximately $85 \%$ of MSI-HCRC. In addition, in almost $50 \%$ of MSI-HCRC, another frequently mutated gene, Bax, was found to have frame-shifting mutations in the polyguanine sequence that inactivate Bax and inhibit apoptosis. In addition to Lynch syndrome, MSI is rarely found in polyps. Patients with Lynch syndrome often develop MSICRC due to germline mutations in one of the MMR genes (MLH1, MSH2, MSH6, and PMS2). Mutations in THE MLH1 or MSH2 genes lead to an increased risk of cancer $(70 \%-80 \%)$, whereas mutations in the MSH6 or PMS2 genes lead to a relatively low risk of cancer development (25\%-60\%), and sporadic MSICRC often show loss of MMR activity due to the silencing of MLH1 by abnormal DNA methylation[19]. In addition, the absence of colon cancer in individuals with MMR gene double-allelic germline mutations suggests that lack of MMR activity is insufficient to trigger polyp formation, according to modeling studies. It is well known that sporadic MSICRC is associated with the serrate tumor formation pathway and usually carries the BRAFV600E mutation, whereas Lynch syndrome is mutated by BRAF lacking mutation in the MMR gene germline[20]. Clinically, BRAF mutated colon cancer patients are associated with poorer prognosis and overall survival (OS) compared with BRAF wild-type disease. Studies have shown that CRC patients with BRAF mutation have the worst OS compared to patients with RAS (KRAS and NRAS) mutations[21]. A recent meta-analysis of 1164 patients with non-metastatic MSI-H showed that the BRAFV600E mutation was associated with the worst OS, but not with disease recurrence[22]. Another meta-analysis of patients undergoing hepatic metastasis resection showed that BRAF mutated metastatic colon cancer had the worst OS after metastatic resection[23]. Similarly, other studies in colon cancer patients have shown that non-V600EBRAF mutated tumors are present in younger populations, low-grade tumors, 
and have significantly longer median OS than V600EBRAF mutated and BRAF wild-type patients[24].

\subsection{CpG Island Methylator Phenotype (CIMP) Pathway}

DNA methylation is the addition of methyl groups to cytosine at the 5' position and is catalyzed by DNA methyltransferase through covalent bonds within CG dinucleotide sequences in the promoter region, known as $\mathrm{CpG}$ transcription[25]. In normal cells, most $\mathrm{CpG}$ sites are heavily methylated, while $\mathrm{CpG}$ islands, usually located in the gene promoter region, are not methylated. However, after the occurrence of cancer, hypermethylation in the promoter region may lead to inactivation of tumor suppressor genes, while overall hypomethylation is associated with genomic instability and chromosomal aberrations[26]. Epigenetic instability in colon cancer is manifested by hypermethylation of loci containing CpG islands, often accompanied by overall DNA hypomethylation. Changes in methylation patterns can affect almost all signaling pathways, including TP53, TGF $/$ SMAD, Wnt, NOTCH and receptor tyrosine kinases, which are involved in cell cycle regulation, transcriptional regulation, DNA stability, apoptosis, intercellular adhesion, angiogenesis, cell invasion and metastasis[27]. Many genes have been identified as methylated and silencing in colon cancer, and some common methylated genes include APC, MLH1, MGMT, SFRP1, SFRP2, CDKN2A, TIMP3, VIM, SEPT, $\mathrm{CDH} 1$, and HLTF. In addition, there is a unique subset of colon cancer called $\mathrm{CpG}$ island methylated phenotype (CIMP)[28]; CIMP tumors often carry BRAFV600E mutations, and CIMP is further subdivided into CIMP2, CIMP- LOW, and CIMP- High based on combined genetic and epigenetic instability. DNA methylation analysis showed that about $20 \%$ of colon cancers were CIMP tumors; CIMP tumors were associated with age, female sex, proximal colon location, and mutations in MSI, KRAS, and BRAF. The most common CIMP tags are MLH1, P16, MINT1, MINT2, and MINT31. Additional markers include CACNA1G, CRABP1, IGF2, NEUROG1, RUNX3, SOCS1, HIC1 and IGFBP3. Although up-regulated expression of DNA methyltransferase (DNMT3B or DNMT1) is associated with CIMP, the underlying mechanisms promoting CIMP remain unknown [29]. One possible underlying mechanism is based on silencing of barriers that inhibit methylation in normal unmethylated $\mathrm{CpG}$ islands. Another suggested mechanism is that changes in chromatin structure and histone modification status of histone $\mathrm{H} 3$ lead to the detection of abnormal DNA methylation at loci where such changes are obtained[30]. Although a tumor-susceptibility gene, PTEN, shows a reduced methylation rate, the gene in TWIST1CRC is silenced by promoter methylation.

\subsubsection{EGFR-KRAS-BRAF Pathway}

Epidermal growth factor receptor (EGFR) is a member of ErbB/HER family. ErbB1 (EGFR/HER1), ErbB2 (NEU/HER2), ErbB3 (HER3), and ErbB4 (HER4). EGFR pathway activation triggers several downstream intracellular signaling pathways, including RAS/RAF/MEK/ERK, PI3K/AKT, and JAK/STAT3 pathways that regulate cell growth, survival, and migration[31-32]. EGFR dysregulation is present in a variety of cancers, including colon cancer; Activation of EGFR induces ras-Raf activation, which leads to phosphorylation of mitogen-activated protein kinases (MAPK or MEK) and activation of extracellular signal-associated kinases (ERK).MAPK pathway includes KRAS and BRAF.RAS/RAF/MAPK pathway, the activation of MAPK includes RAS, RAF and MEK, and is involved in regulating cell proliferation, differentiation, apoptosis and senescence. RAS cascades through phosphoinositol kinase (PI3K) and RAF to stimulate signals.PI3K activation inhibits apoptosis and RAS activation causes cell proliferation, thereby promoting cell survival and tumor invasion and metastasis[33]. KRAS, along with NRAS and HRAS, are oncogenes belonging to the RAS family, which are usually mutated in sporadic colon cancer and are associated with poor prognosis. BRAF, on the other hand, is a member of the RAF serine/threonine kinase family and regulates cell response to growth signals through the Ras-Raf-Map kinase pathway[34]. Braf-activated mutations account for about 5-10\% of metastatic colon cancer, but are rare in the form of Lynch syndrome. Most BRAF mutations include the hot spot mutation V600E (Val600Glu) and have been associated with poor prognosis in CRC patients[35]. Angiogenesis, the development of new blood vessels, is involved in the genesis, growth and metastasis of tumors and involves a number of factors including vascular endothelial growth factor (VEGF). VEGF levels and VEGFR activity are enhanced and associated with poor prognosis in colon cancer, and elevated VEGF levels have been observed in the early stages of colorectal tumors (adenomas). It is significantly elevated in advanced stages of cancer (metastasis). Abnormal KRAS and TP53 and COX-2 expression modulate changes in VEGF-VEGFR activity, thereby promoting cancer growth and migration.

\subsubsection{Notch Pathway}

Notch signaling is composed of transmembrane receptors (Notch1-4), ligands $\delta$-like1, 3, 4, and JagGED-1, as well as JagGED-2, intraconuclear binding proteins and target genes. Notch receptor signaling is initiated by binding to Jagged or $\delta$ like ligands expressed by neighboring cells.In colon cancer, these ligands are produced by stromacell cells, specifically tumor endothelial cells. And ligand binding, depolymerization of Notch receptors are plain-metalloproteinases (ADAM), is then across a membrane structure in the domain of gamma secretase compound cutting, lead to Notch cellular structure domain (notchintracellulardomain, NICD) released from membrane and translocation to the nucleus.Nuclear NICD combined with CSL complex objects then (C-promoterbindingprotein-1/Suppressor of Hairless /Lag1, CBF1-Su(H)-Lag1) to stimulate Notch the expression of target genes, leading to cell proliferation to strengthen (MYC, CCND1), survival (BCL2, NF-kB) and inhibition of cell differentiation (Hes1)[36] Studies have found that Notch 1 and Notch 2 are highly expressed and low expressed in CRC, respectively, and the degree of increase and decrease of Notch 1 and Notch 2 is positively correlated with the pathological stage and malignant degree of CRC, and Notch 1 is highly expressed and Notch 2 is low expressed. They are associated with poor prognosis, which can be used to evaluate the condition and prognosis of colon cancer patients according to their expression level. Kamstrup et al.[37] found that activation of notch-1 signaling pathway can make crypt cells in a state of low differentiation and promote stem cell 
proliferation. Zhang et al.[38] found an increased apoptosis rate of colon cancer cells after Notch gene knockout, and Notch high expression can promote the proliferation of colon cancer cells. Notch expression in coloncancer-initiation cell (CC-IC) was significantly higher than that in normal coloncancer cells. Notch signaling pathway is highly activated in CCSC, and blocking signal transduction of this pathway will affect CCSC metastasis and angiogenesis. Notch signaling is inhibited by two main types of Notch inhibitors: gamma-secretase inhibitors (GSI) and monoclonal antibodies targeting Notch receptors or ligands. GSI, such as DAPT (GSI-IX) inhibitor, inhibited cell proliferation and down-regulated mRNA and protein expressions of ALDH1, Notch-4 and DLL1 in colon cancer cell line HT-29.This may be achieved by lowering CSC characteristics and down-regulating Notch signaling pathway receptor and ligand expression, but its effect has serious side effects and can only be used in adjuvant chemotherapy. Notch inhibitors can be used alone or in combination with chemotherapy drugs for the treatment of cancer and its recurrence. For example, combination with Honokiol can target the expression of Notch-1 and JagGED-1, thereby reducing the expression level of HECS-1, thereby inhibiting the proliferation of CCSC. Rhythm protein homologue 3 (periodcircadianproteinhomolog3, these forms) as a double pathway inhibitor, crossing expression can reduce Notch and Wnt pathways of Notch-1 receptor ligands, Jagged-1, beta serial protein, c-Myc and Lgr5 protein level, Thus enhancing the inhibitory effect of 5-FU on CCSC[39]. In summary, Notch inhibitors can inhibit the proliferation of cancer cells or be used to combat drug resistance in cancer, which could be a novel treatment for cancer. In addition, the combination of Notch and other signaling pathways, such as Wnt and $\mathrm{Hh}$, is also important for cancer treatment, suggesting that the study of cross-substances between different signaling pathways could lead to new strategies for cancer treatment.

\section{Conclusions}

The occurrence and development of colon tumors is a complex biological process, which requires the participation of many genes, proteins and signaling pathways. With the continuous improvement of research, it is necessary to find highly specific tumor markers, further identify and screen out corresponding targets, and then target radical cancer, providing a new idea for the targeted treatment of colon cancer. Studies on colon cancer inhibitors mainly focus on their signaling pathways, and for the status of signaling pathway inhibitors, it is important to strengthen the combination of targeted inhibitors and chemotherapy drugs, so as to reduce the drug resistance of colon cancer and improve the therapeutic effect. In addition, the development of combination inhibitors between signaling pathways is also spurring great vitality. Although many mechanisms of specific markers of colon cancer and their pathway inhibitors are still unclear and need further exploration, both of them, as emerging treatments for colon cancer, will play an important role in the treatment of colon cancer in the future.

\section{References}

[1] Freddie B, B S, M S, et al. Global cancer statistics 2018: GLOBOCAN estimates of incidence and mortality worldwide for 36 cancers in 185 countries[J]. CA: A Cancer Journal for Clinicians, 2018, 68(6): 394-424.

[2] Konda K, Konishi K, Yamochi T, et al. Distinct molecular features of different macroscopic subtypes of colorectal neoplasms[J]. PLo S One, 2014, 9: e103822.

[3] Marzieh A, Isabelle S, Mark J, et al. Global trends in colorectal cancer mortality: projections to the year 2035[J]. International journal of cancer, 2019, 144(12): 2992-3000.

[4] Fearon ER. Molecular genetics of colorectal cancer. Annu Rev Pathol. 2011; 6: 479-507.

[5] Tang AH, Cheng KH, Wong AS.et.al. Current and future molecular diagnostics in colorectal cancer and colorectal adenoma. World J. Gastroenterol. 2014; 20: 3847-3857.

[6] Sebio A, Kahn M, Lenz HJ. The potential of targeting Wnt $/ \beta$-catenin in colon cancer. Expert Opin Ther Targets, 2014, 18: 611-5.

[7] $\mathrm{Cao} \mathrm{H,} \mathrm{Xu} \mathrm{E,} \mathrm{Liu} \mathrm{H,} \mathrm{et} \mathrm{al.} \mathrm{Epithelial-mesenchymal}$ transition in colorectal cancer metastasis: a system review. Pathol Res Pract, 2015, 211: 557-69.

[8] Vermeulen L, De Sousa E, Melo F, et al. The developing cancer stem-cell model: clinical challenges and opportunities. Lancet Oncol, 2012, 13 :e83-9.

[9] Picco G, Petti C, Centonze A, et al. Loss of AXIN1 drives acquired resistance to WNT pathway blockade in colorectal cancer cells carrying RSPO3 fusions. EMBO Mol Med, 2017, 9: 293-303.

[10] Chen Y, Rao X, Huang K, et al. FH535 inhibits proliferation and motility of colon cancer cells by targeting Wnt $/ \beta$-catenin signaling pathway. J Cancer, 2017, 8: 3142-53.

[11] Vogelstein B, Fearon ER, Hamilton SR, et al. Genetic alterations during colorectal-tumor development. N. Engl. J. Med. 1988; 319: 525-532.

[12] Liu Y, Bodmer WF. Analysis of P53 mutations and their expression in 56 colorectal cancer cell lines. Proc. Natl. Acad. Sci. USA. 2006; 103: 976-981.

[13] Colussi D, Brandi G, Bazzoli F, et al. Molecular Pathways Involved in Colorectal Cancer: Implications for Disease Behavior and Prevention. Int. J. Mol. Sci. 2013; 14: 16365-16385.

[14] Zhang J, Su G, Lin Y, et al. Targeting cyclin-dependent kinases in gastrointestinal cancer therapy. Discov. Med. 2019; 27: 27-36.

[15] Jones RG, Plas DR, Kubek S, et al. AMP-activated protein kinase induces a p53-dependent metabolic checkpoint. Mol. Cell. 2005; 18: 283-293.

[16] Park GB, Jin DH, Kim D. Sequential treatment with celecoxib and bortezomib enhances the ER stress-mediated autophagy-associated cell death of colon cancer cells. Oncol. Lett. 2018; 16: 4526-4536.

[17] Mazelin L, Bernet A, Bonod-Bidaud C, et al. Netrin-1 controls colorectal tumorigenesis by regulating apoptosis. Nature. 2004; 431: 80-84.

[18] Ritterhouse LL, Wu EY, Kim WG, et al. Loss of SMAD4 protein expression in gastrointestinal and extra-gastrointestinal carcinomas. Histopathology. 2019; 75: 546-551.

[19] Kaiser JC, Meckbach R, Jacob P. Genomic Instability and Radiation Risk in Molecular Pathways to Colon Cancer. PLoS ONE. 2014; 9: 1-12.

[20] Frouws MA, Reimers MS, Swets M, et al. The Influence of BRAF and KRAS Mutation Status on the Association 
between Aspirin Use and Survival after Colon Cancer Diagnosis. PLoS ONE. 2017; 12: 1-12.

[21] Wang Y, Loree JM, Yu C, et al. Distinct impacts of KRAS, NRAS and BRAF mutations on survival of patients with metastatic colorectal cancer. J. Clin. Oncol. 2018; 36: 3513.

[22] Manthravadi S, Sun W, Saeed A, Prognostic impact of BRAF V600E mutation in patients with non-metastatic colorectal cancer with microsatellite instability: A systematic review and meta-analysis. J. Clin. Oncol. 2018; 36: 3597.

[23] Tosi F, Magni E, Amatu A, et al. Effect of KRAS and BRAF Mutations on Survival of Metastatic Colorectal Cancer After Liver Resection: A Systematic Review and Meta-Analysis. Clin. Colorectal Cancer. 2017; 16: e153-e163.

[24] Shimada Y, Tajima Y, Nagahashi M, et al. Clinical Significance of BRAF Non-V600E Mutations in Colorectal Cancer: A Retrospective Study of Two Institutions. J. Surg. Res. 2018; 232: 72-81.

[25] Wang Y, Liu D, Jin X, et.al. Genome-wide characterization of aberrant DNA methylation patterns and the potential clinical implications in patients with endometrial cancer. Pathol. Res. Pract. 2019; 215: 137-143.

[26] Magzoub MM, Prunello M, Brennan K, et al. The impact of DNA methylation on the cancer proteome. PLoS Comput. Biol. 2019; 15: 1-19.

[27] Bastian PJ, Ellinger J, Heukamp LC, et, al. Prognostic Value of $\mathrm{CpG}$ Island Hypermethylation at PTGS2, RAR-beta, EDNRB, and Other Gene Loci in Patients Undergoing Radical Prostatectomy. Eur. Urol. 2007; 51: 665-674.

[28] Puccini A, Berger MD, Naseem M, et al. Colorectal cancer: Epigenetic alterations and their clinical implications. BBA Rev. Cancer. 2017; 1868: 439-448.

[29] Hanon BM, Al-Mohaimen MNA, Mahmood AS, CpG Island Methylator Phenotype (CIMP) Correlation with Clinical and Morphological Feature of Colorectal Cancer in Iraq patients. Pan Arab J. Oncol. 2015; 8: 6-13.

[30] Zee BM, Dibona AB, Alekseyenko AA, et al. The Oncoprotein BRD4-NUT Generates Aberrant Histone Modification Patterns. PLoS ONE. 2016; 11: 1-16.

[31] Roskoski R. Small molecule inhibitors targeting the EGFR/ErbB family of protein-tyrosine kinases in human cancers. Pharmacolog. Res. 2019; 139: 395-411.

[32] Wang Z. ErbB Receptors and Cancer. Methods Mol. Biol. 2017; 1652: 3-35.

[33] Zhang X, Tang N, Hadden TJ, et al. FoxO and regulation of apoptosis. Biochim. Biophys. Acta. 2011; 1813: 1978-1986.

[34] Samatar AA, Poulikakos PI. Targeting RAS-ERK signalling in cancer: Promises and challenges. Nat. Rev. Drug Discov. 2014; 13: 928-942.

[35] Caputo F, Santini C, Bardasi C, et al. BRAF-Mutated Colorectal Cancer: Clinical and Molecular Insights. Int. J. Mol. Sci. 2019; 20: 5369.

[36] Ntziachristos P, Lim JS, Sage J, et al. From fly wings to targeted cancer therapies: a centennial for notch signaling. Cancer Cell, 2014, 25: 318-34.
[37] Kamstrup MR, Biskup E, Gjerdrum LM, et al. The importance of Notch signaling in peripheral T-cell lymphomas. Leuk Lymphoma, 2014, 55: 639-44.

[38] Zhang Y, Li B, Ji ZZ, et al. Notch1 regulates the growth of human colon cancers. Cancer, 2010, 116: 5207-18.

[39] Zhang F, Sun H, Zhang S, et al. Overexpression of PER3inhibits self-renewal capability and chemoresistance of colorectal cancer stem-like cells via inhibition of notch and $\beta$-catenin signaling. Oncol Res, 2017, 25: 709-19. 